\title{
Applying "Kennedyism" to lung cancer treatment: Let's take more nodes
}

\author{
Richard Lazzaro, MD, and Byron Patton, MD
}

Feature Editor's Note-The importance of lymph node assessment in the surgical treatment of non-small cell lung cancer cannot be overemphasized, because multiple publications have demonstrated that pathologic nodal stage is the strongest predictor of recurrence and longterm survival. There has been a growing body of literature that suggests a prognostic role for the total number of resected lymph nodes, regardless of their metastatic involvement. Other studies invoke lymph node ratio as a predictor of poor survival. The prevailing theory favors more accurate staging in patents with clinical early stage non-small cell lung cancer. Because total lymph node count can often be confounded by fragmentation at the time of surgery, the total number of lymph node stations sampled may be a better surrogate for outcome. As such, the National Comprehensive Care Network has recommended sampling of at least 3 mediastinal lymph node stations or mediastinal lymph node dissection at the time of pulmonary resection. Furthermore, the National Comprehensive Care Network recommends appropriate sampling of N1 and N2 lymph node stations for sublobar resections unless not technically feasible. The American College of Surgeons Commission on Cancer has implemented a quality measure since autumn 2014 of sampling at least 10 regional lymph nodes during surgery for stage I or II non-small cell lung cancer. This quality measure has not been widely adopted by many of the large-volume lung cancer institutions.

In this invited expert opinion, Lazzaro and Patton construct an argument for more lymph node sampling during lung cancer surgery and highlight studies that demonstrate improved cancer-specific survival with increasing numbers of lymph nodes. They bring attention to publications that query large national databases (spanning data collected through the past few decades) and expose a sobering reality

From the Department of Cardiothoracic Surgery, Lenox Hill Hospital, New York, NY. Disclosures: Authors have nothing to disclose with regard to commercial support.

Received for publication Oct 28, 2019; revisions received Oct 28, 2019; accepted for publication Nov 17, 2019; available ahead of print Jan 31, 2020.

Address for reprints: Richard Lazzaro, MD, 130 E 77th St, New York, NY 10075 (E-mail: rlazzaro@northwell.edu).

JTCVS Techniques 2020;1:114-6

2666-2507

Copyright (C 2019 The Authors. Published by Elsevier Inc. on behalf of The American Association for Thoracic Surgery. This is an open access article under the CC BY-NCND license (http://creativecommons.org/licenses/by-nc-nd/4.0/).

https://doi.org/10.1016/j.xjtc.2019.11.006

that lymph node assessment during lung cancer surgery has been unsatisfactory, particularly when sublobar resection is implemented. If adoption of routine mediastinal lymph node dissection during lung cancer surgery has not been implemented in your practice by virtue of the American College of Surgeons Oncology Group Z0030 trial, the culture of systemic lymph node sampling should be mandatory and promulgated in thoracic surgery training programs.

\section{Michael Lanuti, MD}

It is estimated that 228,150 patients will have lung cancer diagnosed and that 142,670 people will die of lung cancer in the United States in 2019. More than 50\% of patients with lung cancer present with distant disease, precluding curative surgery and resulting in an overall 5-year survival approximating $19 \% .{ }^{1}$ Early detection, anatomic resection, and meticulous staging through lymph node evaluation are paramount to improving survival. In addition, minimally invasive lung resections can maximize therapeutic impact without compromising the extent and adequacy of the cancer operations. The thoracic surgeon's judgment and execution are of high importance for good outcomes in an aging population. 
Despite advances in surgical techniques, nonsurgical treatment options will inevitably challenge the current criterion standard-surgical resection with sufficient lymph node removal. It is therefore imperative to revisit who we are and what we do, not as individuals but collectively as a group. From this, we should ask not what patients with lung cancer can do for us but what can we do for patients with lung cancer.

The first step in improving survival is early detection, because advanced disease is so rarely curable. Lung cancer screening has the potential for a $20 \%$ reduction in lung cancer mortality, but it remains underutilized and is performed in fewer than $4 \%$ of eligible patients in the United States. ${ }^{2}$ Currently, $24 \%$ of older patients with lung cancer in the United States present with stage I disease.

Once a lung cancer has been identified on imaging, the presence or absence of nodal disease is often important in determining treatment and prognosis. Little and colleagues ${ }^{4}$ reported on 11,668 patients who underwent initial surgical management of lung cancer. Mediastinoscopy was used preoperatively in $27.1 \%$ of patients; during surgery $57.8 \%$ of patients had mediastinal nodes sampled or resected, with the remaining $42.2 \%$ having no mediastinal nodes removed. ${ }^{4}$ Lymph node sampling versus dissection continues to be studied, with strong proponents of each view. Darling and colleagues ${ }^{5}$ reported the results of the American College of Surgeons Oncology Group (ACOSOG) Z0030 trial, which revealed no difference in either disease-free or overall survival when comparing mediastinal lymph node sampling (MLNS) with mediastinal lymph node dissection (MLND) for patients with T1 or T2 tumors, provided that rigorous systematic mediastinal node sampling was performed either by mediastinoscopy or intraoperatively. Also of interest, MLND was not found to be associated with an increase in either morbidity or mortality. ${ }^{5,6}$ Murthy's sophisticated review ${ }^{7}$ of the impact of the additional $4 \%$ of patients being upstaged after MLND following MLNS in the ACOSOG Z0030 trial yielded a $0.5 \%$ salvage rate, which may be an anticipated consequence of the MLNS in the ACOSOG Z0030 trial; MLNS is an "order of magnitude greater than that which traditionally accompanies a standard lung cancer resection performed in this country," and the 2 arms may be "similar enough that to be statistically indistinguishable," resulting in the negative result. MLND not only has the potential for more accurate staging but can identify the presence of multistation N2 disease as well as skip metastasis. More than 10 lymph nodes were removed in $90 \%$ of the patients in the ACOSOG Z0030 trial with nodes removed from stations $2 \mathrm{R}, 4 \mathrm{R}, 7,8,9$, and $10 \mathrm{R}$ on the right, and stations $4 \mathrm{~L}$, $5,6,7,8,9$, and $10 \mathrm{~L}$ on the left. ${ }^{8}$

It is clear that extensive systematic lymph node sampling for $\mathrm{T} 1$ or $\mathrm{T} 2$ tumors can yield survival data similar to that associated with MLND, but is adequate lymph node sampling or dissection being performed currently? Have we been able to address the concerns raised by Little and colleagues ${ }^{4}$ with $42.2 \%$ of patients undergoing lung resections having no mediastinal lymph nodes removed during lung resection? Bendzsak and coworkers ${ }^{9}$ evaluated the impact of guidelines for invasive mediastinal staging (IMS) use and the effect on nodal sampling and the final stage associated with lack of IMS. In that study, 242 patients underwent lung resection for non-small cell lung cancer: of these, 140 patients received preoperative IMS and 102 patients did not receive preoperative IMS. Overall, staging practices were concordant with IMS guidelines for $85 \%$ of the patients. Although the institution's experience yielded higher rates of lymph node sampling than those reported in the literature, they noted that patients without preoperative IMS had overall lower rates of intraoperative nodal sampling, which may have led to a "considerable number of potentially understaged patients."

Querying the National Cancer Database, Odell and colleagues ${ }^{10}$ found that the American College of Surgeons commission on cancer quality measure, sampling at least 10 lymph nodes during surgery, had been attained in only $23.7 \%$ of cases from 1998 to 2011 . Failure to achieve this quality standard resulted in significantly worse survival (hazard ratio, 1.18; 95\% confidence interval, 1.16-1.2). The effect of a more thorough lymph node evaluation as measured by number of lymph nodes has been supported by previous studies, including a retrospective review by Ludwig and coworkers ${ }^{11}$ of the Surveillance, Epidemiology, and End Results database from 1990 to 2000. The effect of number of lymph nodes resected in 16,800 patients who underwent lung resection and found to be node negative was reported in 2005. Ludwig and coworkers ${ }^{11}$ noted a statistically significant increase in survival when more than 4 lymph nodes were removed. There was an incremental increase in lung cancer-specific survival in patients with 5 to 8 lymph nodes resected (12\%), a further increase in survival for those with 9 to 12 nodes resected $(15 \%)$, and an even further increase in survival with 13 to 16 nodes removed (26\%). There was no further improvement beyond 16 nodes. Potential explanations include stage purification as well as a therapeutic benefit afforded by a more extensive lymph node dissection.

As more early lung cancers are discovered through increased adoption of screening and other initiatives, discussion of sublobar resection plays a prominent role. Indications for sublobar resection, adequacy of margins (margin to tumor ratio $>1 ; 2 \mathrm{~cm}$ ), and lymph node evaluation are all topics of recent interest. Stiles and colleagues ${ }^{12}$ queried the Surveillance, Epidemiology, and End Results Program Medicare data set and identified 1124 propensity-matched pairs of patients who were at least 66 years old, had stage I non-small cell lung cancer, had tumors no larger than $2 \mathrm{~cm}$, and underwent lobectomy versus 
sublobar resection. Of the patients undergoing lobectomy, $94.7 \%$ had lymph nodes removed, compared with only $52.2 \%$ of patients undergoing sublobar resection. Subset analysis comparing lobectomy with sublobar resection demonstrated that when at least 9 lymph nodes were removed, no differences in survival were seen. Only $38.1 \%$ of the patients in the lobectomy group, however, had at least 9 lymph nodes removed, compared with $5.8 \%$ of the patients who underwent sublobar resection.

The minimally invasive approach to anatomic lung resection is associated with many benefits-lessened pain, bleeding, and complications; better tolerance in the elderly population; and greater likelihood of patients to receive and complete adjuvant therapy-but it is often critiqued for the ability to adequately perform lymph node assessment. In reviewing the Society of Thoracic Surgeons database, Boffa and associates ${ }^{13}$ reviewed 11,531 records of resection (lobectomy or segmentectomy) for clinical stage I primary lung cancers. Propensity matching revealed increased nodal upstaging (N0 to N1) with open approach when compared with video-assisted thoracoscopic surgery $(9 \%$ vs $6.8 \%$; $P=.002)$. Recognizing your own personal experience regarding the thoroughness of operative lymph node evaluation can lead to a "more focused lymph node sampling with VATS [video-assisted thoracoscopic surgical] lobectomy." 14

Adequate lymph node evaluation is a quality measure that maximizes accurate staging and potentially provides an innate therapeutic benefit. This is a standard that should be adhered to by all surgeons for every operation. Despite numerous studies reporting the benefits of lymph node evaluation, data from large national databases confirm that many are still coming up short. Whether MLNS or MLND is performed, the surgeon has an obligation to the patient with lung cancer. Because it remains impossible to determine histopathology on the basis of visual or tactile examination, the time is now to embrace extensive systematic nodal sampling evaluation according to ACOSOG Z0030 recommendations. It remains imperative to develop mentoring workshops and standardized training programs focused on the anatomic landmarks and safe performance of MLND. Each surgeon should obey the dictates of his or her own conscience with regard to the value-added benefit of the performance of a systematic mediastinal lymphadenectomy or MLNS according to ACOSOG Z0030 to ensure best patient outcomes. Remember, ask not what your patient with lung cancer can do for you, ask what you can do for your patient with lung cancer. Or, more directly, let's take more nodes.

\section{References}

1. Siegel RL, Miller KD, Jemal A. Cancer statistics, 2019. CA Cancer J Clin. 2019; 69:7-34.

2. Jemal A, Fedewa SA. Lung cancer screening with low-dose computed tomography in the United States-2010 to 2015. JAMA Oncol. 2017;3:1278-81.

3. Andreano A, Peake MD, Janes SM, Valsecchi MG, Pritchard-Jones K, Hoag JR, et al. The care and outcomes of older persons with lung cancer in England and the United States, 2008-2012. J Thorac Oncol. 2018;13:904-14.

4. Little AG, Rusch VW, Bonner JA, Gaspar LE, Green MR, Webb WR, et al. Patterns of surgical care in lung cancer patients. Ann Thorac Surg. 2005;80:2051-6; discussion 2056.

5. Darling GE, Allen MS, Decker PA, Ballman K, Malthaner RA, Inculet RI, et al. Randomized trial of mediastinal lymph node sampling versus complete lymphadenectomy during pulmonary resection in the patient with N0 or N1 (less than hilar) non-small cell carcinoma: results of the American College of Surgery Oncology Group Z0030 trial. J Thorac Cardiovasc Surg. 2011; 141:662-70.

6. Wang Y, Darling GE. Complete mediastinal lymph node dissection versus systematic lymph node sampling in surgical treatment of non-small cell lung cancer: do we have the answer? J Thorac Dis. 2017;9:4169-70.

7. Murthy SC. Less is more... (more or less...). J Thorac Cardiovasc Surg. 2011; 141:670-2.

8. Darling GE, Allen MS, Decker PA, Ballman K, Malthaner RA, Inculet RI, et al. Number of lymph nodes harvested from a mediastinal lymphadenectomy: results of the randomized, prospective American College of Surgeons Oncology Group Z0030 trial. Chest. 2011;139:1124-9.

9. Bendzsak A, Waddell TK, Yasufuku K, Keshavjee S, de Perrot M, Cypel M, et al. Invasive mediastinal staging guideline concordance. Ann Thorac Surg. 2017; 103: 1736-41.

10. Odell DD, Feinglass J, Engelhardt K, Papastefan S, Meyerson SL, Bharat A, et al. Evaluation of adherence to the commission on cancer lung cancer quality measures. J Thorac Cardiovasc Surg. 2019;157:1219-35.

11. Ludwig MS, Goodman M, Miller DL, Johnstone PA. Postoperative survival and the number of lymph nodes sampled during resection of node-negative non-small cell lung cancer. Chest. 2005; 128:1545-50.

12. Stiles BM, Mao J, Harrison S, Lee B, Port JL, Sedrakyan A, et al. Extent of lymphadenectomy is associated with oncological efficacy of sublobar resection for lung cancer $\leq 2 \mathrm{~cm}$. J Thorac Cardiovasc Surg. 2019;157:2454-65.

13. Boffa DJ, Kosinski AS, Paul S, Mitchell JD, Onaitis M. Lymph node evaluation by open or video-assisted approaches in 11,500 anatomic lung cancer resections. Ann Thorac Surg. 2012;94:347-53; discussion 353.

14. Denlinger CE, Fernandez F, Meyers BF, Pratt W, Zoole JB, Patterson A, et al. Lymph node evaluation in video-assisted thoracoscopic lobectomy versus lobectomy by thoracotomy. Ann Thorac Surg. 2010;89:1730-5; discussion 1736. 